\title{
MAC ADDRESS CLONING TECHNIQUE RESULTS
}

\author{
Md. Abdullah Yusuf Imam \\ Assistant Maintenance Engineer \\ Department Of ICT \\ National University \\ Gazipur-1704, Bangladesh
}

\author{
Mr. Prodip Kumar Biswas \\ Sub-Technical Officer \\ Department Of ICT \\ National University \\ Gazipur-1704, Bangladesh
}

\begin{abstract}
MAC filtering is not an effective control in wireless networking as attackers can eavesdrop on wireless transmissions mode. However MAC filtering is more effective in wired network, since it is more difficult for attacker to identify authorized MAC address. MAC address are uniquely assigned to each LAN card. In this way, Changing MAC address is necessary in network virtualization. It can also be used in the process of exploiting security of a system. In IP networks, the MAC address of an interface is query given the IP address using the Address Resolution Protocol (ARP) for Internet Protocol Version 4 (IPv4) or the Neighbor Discovery Protocol (NDP) for IPv6. ARP and NDP is used to relate IP addresses (OSI layer 3) to Ethernet MAC addresses (OSI layer 2).
\end{abstract}

Keywords- Mac, Ip, Network, Address

\section{HOW TO GET MAC ADDRESS OF WINDOWS VISTA, 7,8,10 USING COMMAND PROMT}

- Click The Start Button.

- In The Run Text Box, Type Cmd. This Will Open The Command Window.

- At the Command Prompt, Type ipconfig /all

- Copy Down The "Physical Address" (The Mac Address). [Figure:1] Shows the result.

* For the Ethernet adapter Wireless Network Connection:

Do the same way \& [Figure:2] shows the result.

For widows 8:

$\checkmark \quad$ Press the Windows Start key to open the Start screen.

$\checkmark \quad$ Type cmd and press Enter to launch the command prompt.

[Figure:3] shows the result.
For windows 10:

$>$ Open the command prompt. Right-click on the Start button and select Command Prompt from the menu.

$>$ Do the same way \& [Figure:4] shows the initial result.

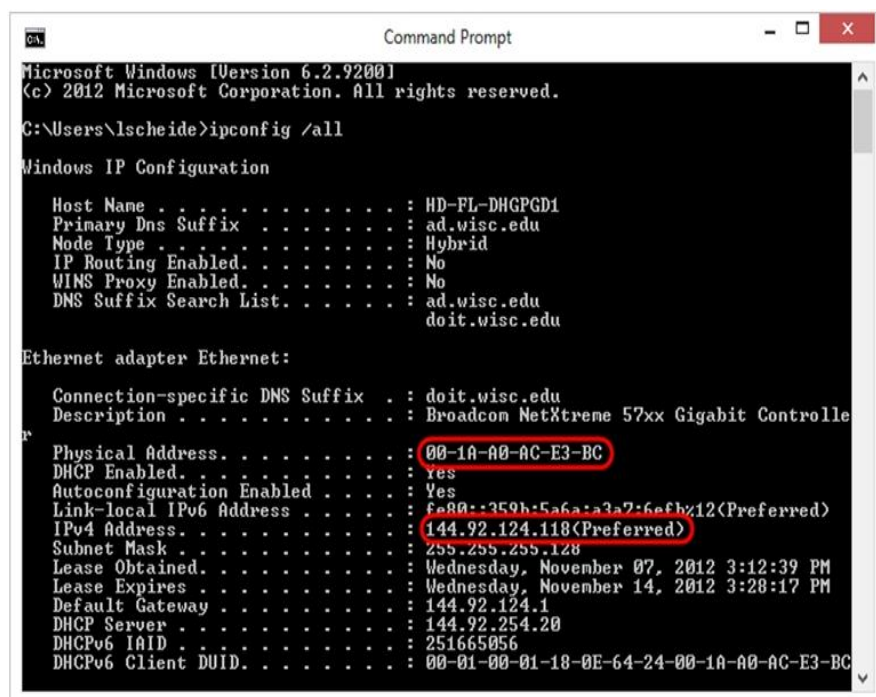

Fig.1. Finding Mac Address

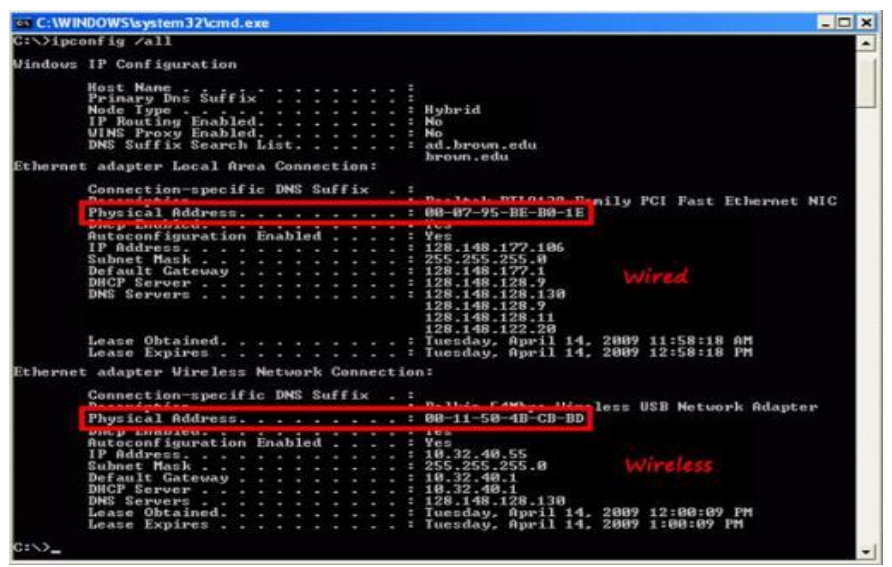

Fig.2. Finding Mac Address of Ethernet adapter 


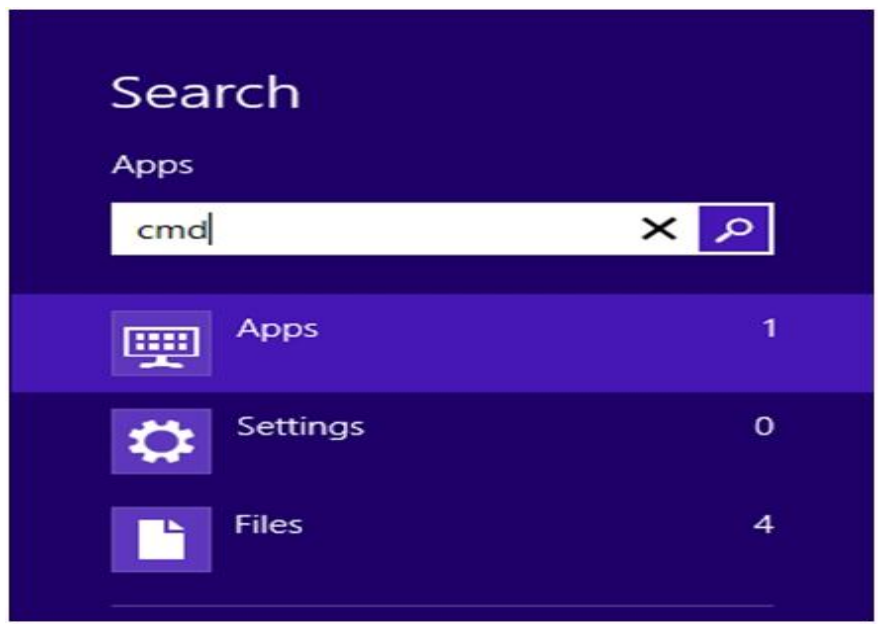

Fig.3. For windows 8

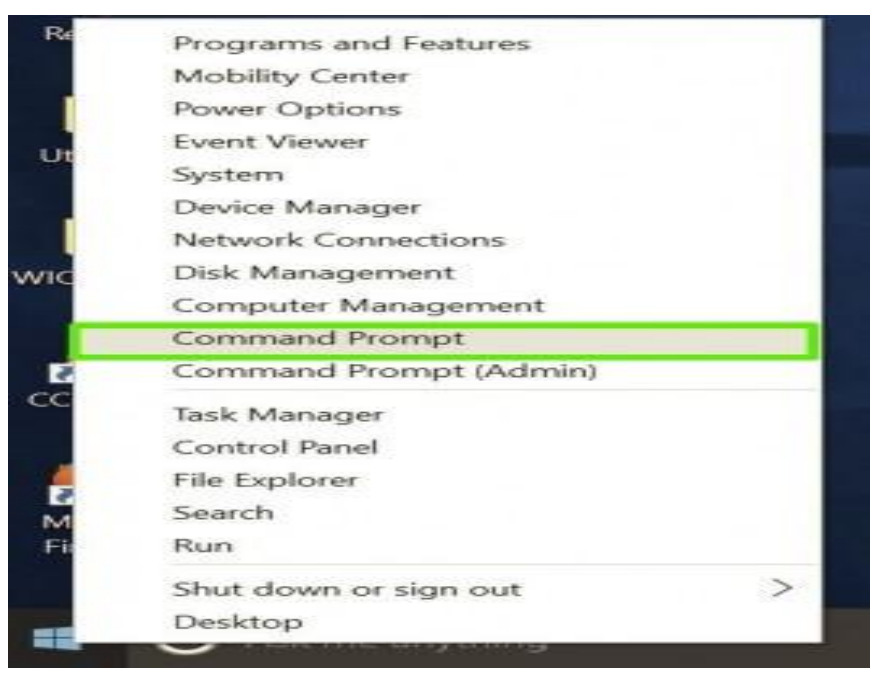

Fig.4. MAC address finding for windows 10

\section{MAC ADDRESS AND IP ADDRESS RELATIONSHIP}

Walter Goralski et al.(2015) in their work emphasize and addressed issues of TCP/IP network uses MAC addresses and IP addresses but for separate purposes. A MAC address remains fixed to the device's hardware while the IP address for that same device can be changed depending on its TCP/IP network configuration policy. Rick Boivie, Nancy Feldman, Yuji Imai, Wim Livens \& Dirk Ooms (November 2007) et al. in their work emphasize and addressed issues of working scope about Media Access Control, which operates at Layer 2 of the OSI model while Internet Protocol operates at Layer 3 of OSI layers. This allows MAC addressing to support other kinds of networks besides TCP/IP.
IP networks manage the connections between IP and MAC addresses using Address Resolution Protocol (ARP).

N. Sinha, R. Oz and S. V. Vasudevan et al. in their work emphasize and addressed issues of while the IP addresses involved indicate the original source and destination, a MAC address is used only on connections from one piece of networking equipment to the next to go.

\section{WILL ANYONE EVER NEEDS TO CHANGE MAC ADDRESS?}

In General, Anyone will not find any cause for changing his MAC address. This is simply a number that identifies the network device that are using and there shouldn't be any reason to change it [2],[3]. However, some internet user may encounters issues with their ISP when they change equipments, such as upgrading their routers or getting a new residential gateway. The ISP has essentially linked the MAC address of the old equipment to the IP. When this equipment changes, so does the MAC address, leaving a user unable to access the internet [3][4].

This is when MAC address cloning comes into focus. When this happens, the ISP links the new hardware's IP to the old MAC address so that connectivity is maintained as regular. If, anyone is choosing to update his own equipment, a quick call to ISP can help clear up any service issues caused by new MAC address that do not match. This may include providing with troubleshooting steps to change router or equipment settings of MAC address to be cloned. However, other than these instances, your MAC address is fixed and will not need to be changed.

Most ISP assign their IP binding with equipments MAC [5]. If the MAC address of a device is 00-11-22-33-44-55 and connect to ISP, the DHCP server records the MAC address and assigns an IP address against it [6],[7],[8]. If it disconnect from the ISP, the device loses IP address. The next time when the device is connected, the DHCP server sees the device's MAC address, looks to see if it has assigned an IP address before. If it has and the lease time has not expired, it will most likely to give the same IP address that had before disconnecting.

\section{PROBLEMS IN GENERAL MAC ADDRESSING}

1. Occasionally, manufacturers don't ensure they are unique, so multiple network interfaces in one system can in fact have the same MAC address [9],[10].

2. In many network interfaces, the MAC address can be set by software, meaning whatever the original MAC address, it can 


\section{International Journal of Engineering Applied Sciences and Technology, 2019 \\ Vol. 4, Issue 1, ISSN No. 2455-2143, Pages 1-5 \\ Published Online May 2019 in IJEAST (http://www.ijeast.com)}

be overridden. So the uniqueness on which anyone may relies is not completely unreliable [11].

\section{CAVEATS TO CHANGING MAC ADDRESS BY SOFTWARE}

Changing MAC address is only temporary basis. Once reboot the machine, the operating system reflects the physical MAC address burnt in one's network card and not the MAC address which was set [12].

\section{NEW MAC ADDRESS GENERATION TO CHANGE MAC ADDRESS}

There are actually 4 sets of Locally Administered Address Ranges that can be used on network without fear of conflict to each other, assuming no one else has assigned these on any network:

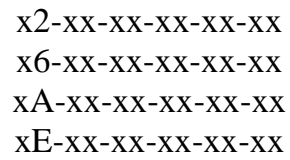

Replacing x with any hex value [13],[14].

\section{MAC ADDRESS CAN BE CHANGEABLE OR CLONE WITHOUT ANY SOFTWARE PERMANENTLY}

Method:1:-

$>$ Open Network and Sharing Center, choose Change Adapter Settings on the left.

$>$ Right click on your wireless adapter and choose properties.

$>$ Make sure the Networking tab is selected (should be by default) and choose Configure.

$>$ Click the Advanced tab.

$>$ In the Property window, choose Network Address and select value \&insert your desired mac without ". ".

$>$ Press ok.

$>$ To width draw given network address: Select not present.

Method:2:-

$\checkmark \quad$ First, open the Device Manager. On Windows 8 and 10 , press Windows $+X$, and then click "Device Manager" on the Power User menu. On Windows 7, press the Windows key, type "Device Manager" to search for it, and then click the "Device Manager" entry. The Device Manager app will look the same no matter which version of Windows you're using.

$\checkmark$ In Device Manager, under the "Network adapters" section, right-click the network interface you want to modify, and then select "Properties" from the context menu.

$\checkmark$ In the properties window, on the "Advanced" tab and select the "Network Address" entry in the "Property" list. If you don't see this option, then your network driver doesn't support this feature.

$\checkmark$ Enable the Value option and type your desired MAC address without any separating characters-don't use dashes or colons. Click "OK" when you're done,[Figure 5,6] shows the details.

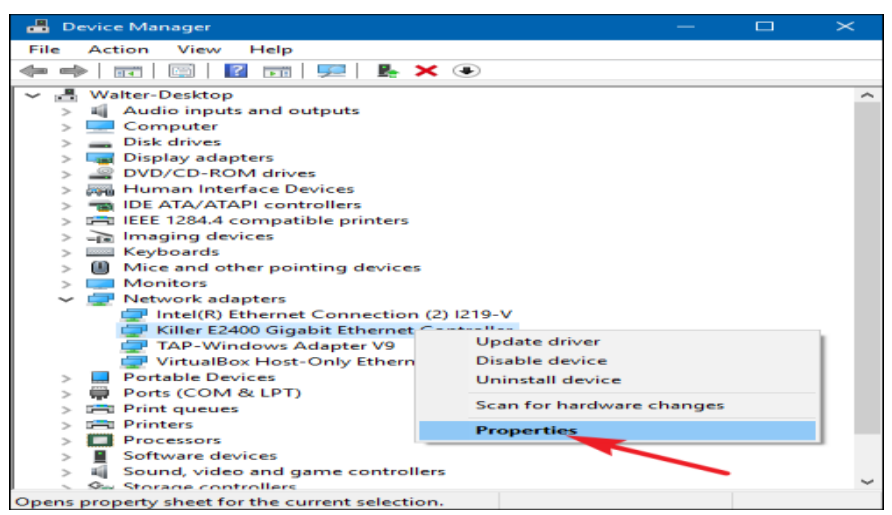

Fig.5. MAC address change options

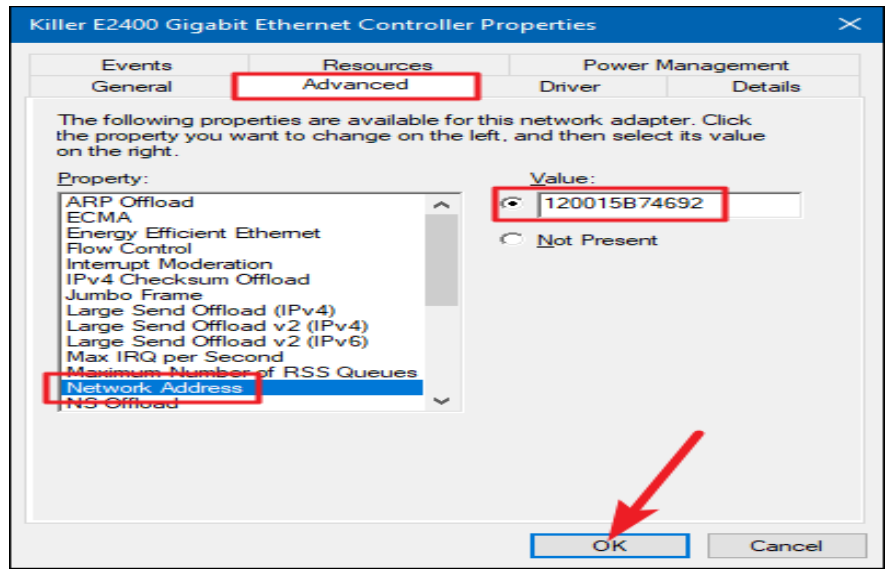

Fig.6. MAC address change policy

\section{MAC ADDRESS CHANGING SOFTWARE}

Noor mohammadpour, Mohammad, Raghavendra, Cauligi (16 July 2018) et al. in their work emphasize and addressed issues 


\section{International Journal of Engineering Applied Sciences and Technology, 2019 Vol. 4, Issue 1, ISSN No. 2455-2143, Pages 1-5 \\ Published Online May 2019 in IJEAST (http://www.ijeast.com)}

of use third-party tools to change your MAC address: Technitium MAC address changer is a free $3^{\text {rd }}$ party software which works well with all versions of windows Operating system right from windows XP to Windows 10. After running it, the software will automatically detect all the network adapters installed in the system and provide options to change the MAC address of active network adapter.

Noor mohammadpour, Mohammad, Srivastava, Ajitesh, Raghavendra, Cauligi (2018) ) et al. in their work emphasize and addressed issues- if anyone wants to use a MAC address changer to change MAC address on Windows 10 , there are few tools that can use. One of these tools is Technitium MAC Address Changer. To use this tool to change MAC address, following are the steps:

1. Need to download Technitium MAC Address Changer.

2. Once downloaded and installed this tool, needed to run it.

3. When Technitium MAC Address Changer starts, it shows the list of all network adapters available.

4. Need to select the network adapter to change and in the CHANGE MAC address section need to enter a new MAC address. It need to be remembered to use a hexadecimal value. If anyone wishes to make this step faster,can just click Random MAC Address button that will generate a random MAC address for him.

5. Click Change Now! button and MAC address should be changed.

6. OPTIONAL: To restore default MAC address just click the Restore Original button,[Figure 7] shows the reflections.

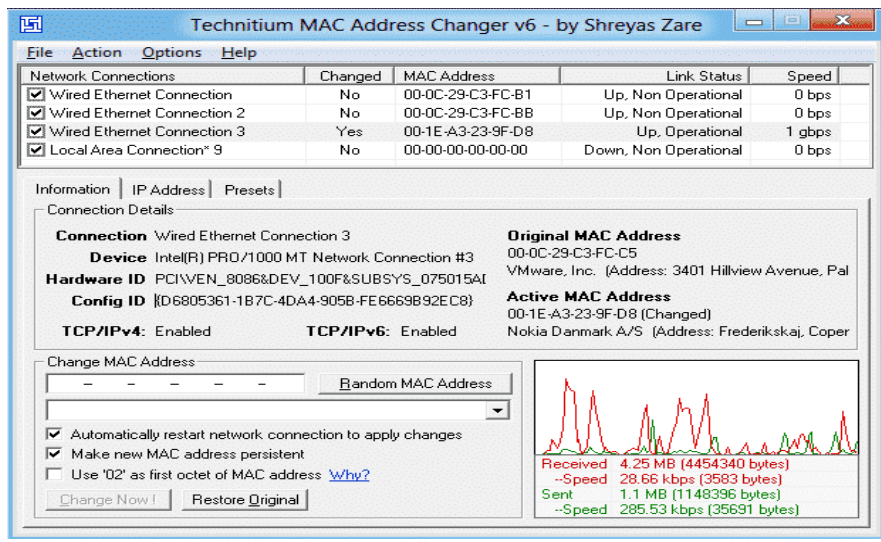

Fig.7.Third Party Software to change Mac address

\section{CONCLUSION}

Some internet service providers bind their service to a specific MAC address; if the user then changes their network card or intends to install a router, the service won't work anymore. Changing the MAC address of the new interface will solve the problem.

\section{REFERENCES}

[1] Walter Goralski (2015), The Illustrated Network: How TCP/IP Works in a Modern Network (PDF).Morgan Kaufmann. p. 26.ISBN 9780123745415 .

[2] Lawrence Harte, Introduction to Data Multicasting, Althos Publishing 2008.

[3] M. Noor mohammadpour; et al. (July 10, 2017). "DCCast: Efficient Point to Multipoint Transfers Across Datacenters". USENIX. Retrieved July 26, 2017.

[4] Rick Boivie; Nancy Feldman; Yuji Imai; Wim Livens \& Dirk Ooms (November 2007). "Explicit Multicast (Xcast) Concepts and Options".Retrieved May 25, 2013.

[5] N. Sinha, R. Oz and S. V. Vasudevan, "The statistics of switched broadcast", Proceedings of the SCTE 2005 Conference on Emerging Technologies, Tampa, FL, USA, January 2005

[6] MichaelMitzenmacher; Andréa W. Richa; Ramesh Sitaraman. "The Power of Two Random Choices: A Survey of Techniques and Results". Section "Randomized Protocols for Circuit Routing". p. 34.

[7] M. Noor mohammadpour; C. S. Raghavendra, (2018). "Poster Abstract: Minimizing Flow Completion Times using Adaptive Routing over Inter-Datacenter Wide Area Networks".

[8] M. Noormohammadpour; C. S. Raghavendra. (2018). "Minimizing Flow Completion Times using Adaptive Routing over Inter-Datacenter Wide Area Networks".

[9] Jonne Zutt, Arjan J.C. van Gemund, Mathijs M. de Weerdt, and Cees Witteveen (2010). Dealing with Uncertainty in Operational Transport Planning. In R.R. Negenborn and Z. Lukszo and H. Hellendoorn (Eds.) Intelligent Infrastructures, Ch. 14, pp. 355382. Springer.

[10] Matthew Caesar and Jennifer Rexford. BGP routing policies in ISP networks. IEEE Network Magazine, special issue on Interdomain Routing, Nov/Dec 2005.

[11]Neil Spring, Ratul Mahajan, and Thomas Anderson. Quantifying the Causes of Path Inflation.Proc. SIGCOMM 2003.

[12]Ratul Mahajan, David Wetherall, and Thomas Anderson. Negotiation-Based Routing Between Neighboring ISPs.Proc. NSDI 2005. 
[13]Ratul Mahajan, David Wetherall, and Thomas Anderson. Mutually Controlled Routing with Independent ISPs.Proc. NSDI 2007.

[14] Khalidi, Yousef (March 15, 2017). "How Microsoft builds its fast and reliable global network".

[15] Noor mohammadpour, Mohammad; Raghavendra, Cauligi (16 July 2018). "Datacenter Traffic Control: Understanding Techniques and Tradeoffs". Communications Surveys \& Tutorials, IEEE.20 (2): 1492-1525.

[16] Noor mohammadpour, Mohammad; Srivastava, Ajitesh; Raghavendra, Cauligi (2018). "On Minimizing the Completion Times of Long Flows over Inter-Datacenter WAN". Communications Letters, IEEE. 